\title{
Análise da cinética de secagem em camada fina de grãos de lentilha - Lens Culinaris
}

\author{
Analysis of the thin layer drying kinetic of brown lentil grains - Lens Culinaris \\ Análisis de la cinética de secado de capa delgada de granos de lentejas - Lens Culinaris
}

Recebido: 10/08/2021 | Revisado: 15/08/2021 | Aceito: 17/08/2021 | Publicado: 20/08/2021

Eudezia Rodrigues Mangueira ORCID: https://orcid.org/0000-0002-7461-7201 Universidade Federal de Campina Grande, Brasil E-mail: ezia.rodrigues@hotmail.com

Maria de Sousa Leite Filha ORCID: https://orcid.org/0000-0003-4576-3979 Universidade Federal de Campina Grande, Brasil E-mail: marialeitefilha@ hotmail.com

José Jorge da Silva Junior ORCID: https://orcid.org/0000-0003-4497-5689 Universidade Federal de Campina Grande, Brasil E-mail: josejorge_18@hotmail.com

Clenilson Ferreira da Silva ORCID: https://orcid.org/0000-0003-1201-4787 Universidade Federal de Campina Grande, Brasil E-mail: clenilsonfds@hotmail.com

Welington Bezerra de Sousa ORCID: https://orcid.org/0000-0003-0727-3290 Universidade Federal de Campina Grande, Brasil

E-mail: welingtonbezerra@yahoo.com.br

Mário Eduardo Rangel Moreira Cavalcanti-Mata ORCID: https://orcid.org/0000-0001-6919-207X Universidade Federal de Campina Grande, Brasil

E-mail: mcavalcantimata@gmail.com

Antônio Gilson Barbosa de Lima ORCID: https://orcid.org/0000-0003-1691-1872 Universidade Federal de Campina Grande, Brasil E-mail: antonio.gilson@ufcg.edu.br

Marcelo Bezerra Grilo

ORCID: https://orcid.org/0000-0002-6415-5058 Universidade Federal de Campina Grande, Brasil E-mail: marcelogrilo@gmail.com

José Jefferson da Silva Nascimento ORCID: https://orcid.org/0000-0002-2620-6491 Universidade Federal de Campina Grande, Brasil E-mail: jeffpesquisador@gmail.com

Severino Rodrigues de Farias Neto ORCID: https://orcid.org/0000-0001-9826-2110 Universidade Federal de Campina Grande, Brasil

E-mail: severino.rodrigues@eq.ufcg.edu.br

Ana Paula Trindade Rocha ORCID: https://orcid.org/0000-0002-0230-7394 Universidade Federal de Campina Grande, Brasil E-mail: ana_trindade@yahoo.com.br

\begin{abstract}
Resumo
Neste trabalho são analisados diferentes perfis de secagem em camada fina para grãos de lentilha marrom - Lens Culinaris nas temperaturas de $35,45,55$ e $65^{\circ} \mathrm{C}$, na velocidade de $0,8 \mathrm{~m} / \mathrm{s}$ e teor de água de $25 \%$. O objetivo deste trabalho consiste em analisar a cinética de secagem de grãos de lentilha, ajustando os parâmetros observados para as temperaturas de $35,45,55$ e $65{ }^{\circ} \mathrm{C}$, com teor de água inicial de $25 \%$ base úmida, a diferentes modelos matemáticos existentes, além de determinar as propriedades termodinâmicas (entalpia, entropia e energia livre de Gibbs) para o processo. O processo de secagem dos grãos foi realizado no Laboratório de Armazenamento e Processamento de Produtos Agrícolas (LAPPA) do Departamento de Engenharia Agrícola da Universidade Federal de Campina Grande - UFCG. Os grãos utilizados no estudo foram adquiridos no comercio local de Campina Grande - PB. Onde foram analisadas as curvas cinéticas para diferentes modelos matemáticos. Desse modo foi possível observar que o modelo com o melhor ajuste foi o modelo Cavalcanti-Mata.
\end{abstract}

Palavras-chave: Lentilha; Secagem em camada; Modelagem Matemática. 


\begin{abstract}
In this work, different thin layer drying profiles for brown lentil grains - Lens Culinaris at temperatures of $35,45,55$ and $65{ }^{\circ} \mathrm{C}$, at a speed of $0.8 \mathrm{~m} / \mathrm{s}$ and $25 \%$ water content are analyzed. The objective is to analyze the drying kinetics of lentil grains, adjusting the parameters observed for temperatures of $35,45,55$ and $65{ }^{\circ} \mathrm{C}$, with an initial water content of $25 \%$ wet basis, to different mathematical models, in addition to determining the thermodynamic properties (enthalpy, entropy and Gibbs free energy) for the process. The drying process of the grains was performed at the Laboratório de Armazenamento e Processamento de Produtos Agrícolas (LAPPA) of the Agricultural Engineering Department of the Federal University of Campina Grande - UFCG. The grains used in the study were acquired in the local market of Campina Grande - PB. Where the kinetic curves for different mathematical models were analyzed. Thus, it was possible to observe that the model with the best fit was the Cavalcanti Mata model.
\end{abstract}

Keywords: Lentil; Layer drying; Mathematical Modeling.

\title{
Resumen
}

En este trabajo se analizan diferentes perfiles de secado en capa fina para granos de lentejas pardas - Lens Culinaris a temperaturas de $35,45,55$ y $65^{\circ} \mathrm{C}$, a una velocidad de $0,8 \mathrm{~m} /$ sy un contenido de agua del $25 \%$. El objetivo de este trabajo es analizar la cinética de secado de los granos de lentejas, ajustando los parámetros observados para temperaturas de $35,45,55$ y $65^{\circ} \mathrm{C}$, con un contenido inicial de agua del $25 \%$ en base húmeda, a diferentes modelos matemáticos existentes, en Además de determinar las propiedades termodinámicas (entalpía, entropía y energía libre de Gibbs) para el proceso. El proceso de secado de los granos se realizó en el Laboratorio de Almacenamiento y Procesamiento de Productos Agrícolas (LAPPA) del Departamento de Ingeniería Agrícola de la Universidad Federal de Campina Grande - UFCG. Los granos utilizados en el estudio fueron adquiridas en el mercado local de Campina Grande - PB. Donde se analizaron las curvas cinéticas para diferentes modelos matemáticos. Así, se pudo observar que el modelo con mejor ajuste fue el modelo Cavalcanti Mata.

Palabras clave: Lenteja; Secado de capas; Modelo Matematico.

\section{Introdução}

A lentilha é um grão pertencente à espécie Lens esculenta Moench (Brasil, 2009), com nome cientifico de Lens culinaris (Vohra \& Gupta, 2012). É uma das mais antigas leguminosas cultivadas pelo homem (Vieira et al., 2001) originária do sudoeste da Ásia, que vem tornando-se popular devido às pesquisas que relacionam seu consumo a benefícios à saúde. É a quinta leguminosa mais produzida no mundo sendo que seu consumo aumentou cinco vezes nas últimas décadas (Joshi, Timilsena \& Adhikari, 2017), embora o Brasil apresente condições favoráveis para seu cultivo e boa aceitação no mercado, a produção brasileira de lentilha ainda é relativamente pequena, fazendo-se necessária a quase totalidade de importação (Vieira et al., 2001).

A lentilha é uma hortaliça de ciclo anual e está entre as cinco leguminosas mais importantes no mundo (Nascimento, Vieira \& Lima, 2016). Bastante utilizada nos países árabes (FAO 2014). Há grande variedade de lentilhas no mundo, apresentando cada uma sua característica e particularidades (CGC, 2014), os grãos de lentilha apresentam formato de lente, com diâmetros variando entre $2-9 \mathrm{~mm}$, com superfície lisa tendo como principal carboidrato formador da parede celular a arabinose. Os grãos de lentilha podem assumir uma gama de cores (Bhatty, 1990) e são agrupadas de acordo com classificação baseada no tamanho e na coloração do grão (Gupta et al., 2013).

As características físicas dos grãos são relacionadas diretamente a diferentes parâmetros ligados à sua armazenagem e demais procedimentos nela envolvidos, como aeração e prevenção do aparecimento e proliferação de pragas. Pelo correto armazenamento há reflexão positiva nos parâmetros econômicos e nutricionais (Oliveira et al., 2013). A lentilha pode conter mais de $28 \%$ de proteína, sendo este valor dependente de diversos fatores, visto que esse componente é acumulado no grão durante o processo de crescimento e maturação (Roy, Boye \& Simpson, 2010).

O processo de secagem de produtos alimentícios é um dos processos mais utilizados, pois ocorre uma considerável diminuição da atividade de água, o que reduz atividades microbiológicas durante o armazenamento e diminui mudanças física, química e microbiológicas, fazendo com que ocorra o aumento da estabilidade do alimento e aumente seu tempo de conservação (Resende et al., 2008). 
A redução do teor de água para grãos é um processo complexo, pois, ao mesmo tempo que envolve a transferência de calor e massa, é necessário que se mantenha a qualidade e as propriedades físicas dos grãos (Mayor \& Sereno, 2004). Grãos de lentilha danificados durante o processo de secagem resulta em um produto de menor qualidade e consequentemente uma redução no preço do mercado, pois torna o grão passível ao ataque de microrganismos e fungos durante o armazenamento (Tang \& Sokhansanj, 1993).

Diante ao exposto, o objetivo deste trabalho foi analisar a cinética de secagem de grãos de lentilha, ajustando os parâmetros observados para as temperaturas de $35,45,55$ e $65{ }^{\circ} \mathrm{C}$, com teor de água inicial de $25 \%$ base úmida, a diferentes modelos matemáticos existentes, além de determinar as propriedades termodinâmicas (entalpia, entropia e energia livre de Gibbs) para o processo.

\section{Metodologia}

O artigo apresenta um estudo de caso de caráter qualitativo tomando em consideração a afirmação de Martins (2017) que diz que: "O estudo de caso é um tipo de método onde se estuda casos específicos ou que envolvem pequenos grupos, buscando entender como determinados fatores ocorrem" (Martins, 2017, p. 16). Martins (2017) afirma ainda que "para desenvolver uma pesquisa quantitativa é necessário ter variáveis bem definidas e utilizar cálculos estatísticos e/ou interferências" (Martins, 2017, p. 19).

O estudo consiste na análise da cinética de secagem durante o processo de secagem em camada fina dos grãos de lentilha, ajustando os parâmetros observados para as temperaturas estudadas à diferentes modelos matemáticos, além de determinar propriedades termodinâmicas, como, entalpia, entropia e energia livre de Gibbs para o processo.

O processo de secagem dos grãos de lentilha foi realizado no Laboratório de Armazenamento e Processamento de Produtos Agrícolas (LAPPA) do Departamento de Engenharia Agrícola da Universidade Federal de Campina Grande - UFCG. Os grãos utilizados no estudo foram da Lentilha marrom, adquiridos no comercio local da cidade de Campina Grande - PB.

Inicialmente foi determinado o teor de água inicial, realizado pelo método padrão de estufa a $105 \pm 3^{\circ} \mathrm{C}$, por um período de $24 \mathrm{~h}$, utilizando três subamostras de $10 \mathrm{~g}$ de grãos de Lentilha, dispostos em recipientes metálicos conforme descrito pelas Regras para Análise de Sementes (Brasil, 2009). As pesagens das amostras foram realizadas por meio de uma balança analítica (ANALYTICAL Standard - OHAUS) com precisão de 0,0001 g. A porcentagem de teor de água foi calculada em base úmida aplicando-se a Equação 1, sendo o resultado final expresso pela média aritmética das subamostras.

Teor de Água $(\%)=\frac{100(M-m)}{M-t}$

Onde $M=$ massa inicial, massa do recipiente mais a massa dos grãos úmidos; $m=$ massa final, massa do recipiente mais a massa da semente seca e $t=$ tara, massa do recipiente.

Após conhecido o teor de água, foi realizado o processo de secagem e determinação das curvas cinéticas utilizando um secador de leito fixo (Figura 1), disponível no Laboratório de Secagem de Produtos Agrícolas localizado na Universidade Federal de Campina Grande - UFCG, sendo este ajustado nas temperaturas de $35,45,55$ e $65{ }^{\circ} \mathrm{C}$. As amostram dos grãos de lentilha com teor de água inicial de aproximadamente $25 \%$ em base úmida, foram pesadas em uma balança analítica com precisão de 0,001 g em intervalos de tempo regulares de 5, 10,20, 30 e 60 minutos, até que atingisse peso constante, que equivale a determinar o teor de água de equilíbrio para as específicas condições termodinâmicas do ar de secagem. 
Figura 1: Esquema representativo da vista lateral do secador de leito fixo utilizado na secagem dos grãos de Lentilha.

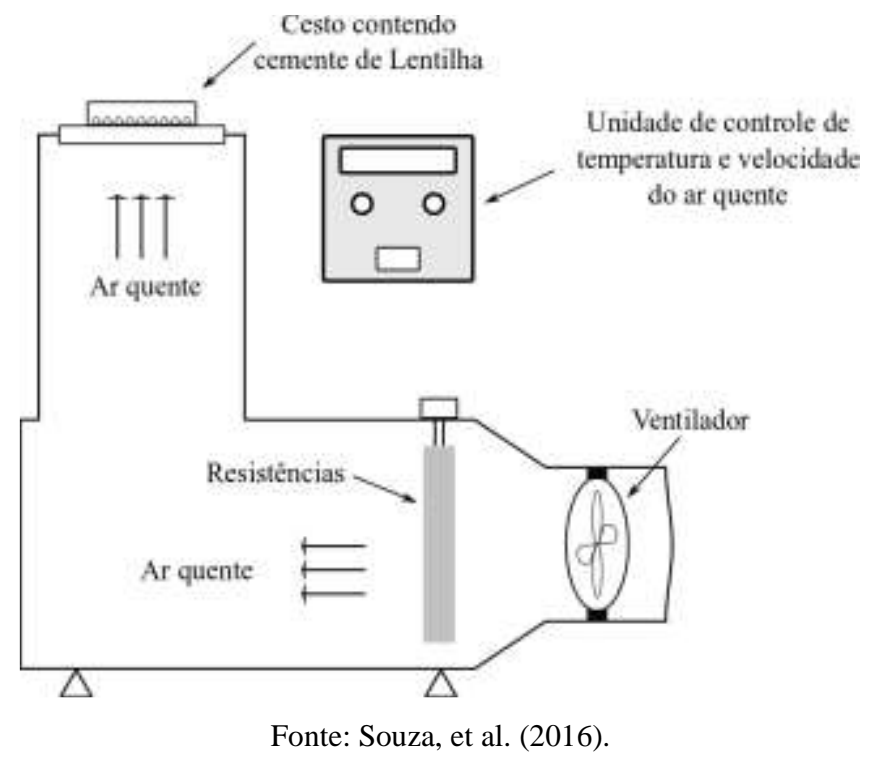

A redução do teor de água foi obtida pelo método gravimétrico (perda de água), conhecendo-se o teor de água inicial do produto até atingir o seu ponto de equilíbrio, no parâmetro adimensional razão de teor de água $(R X)$. Para determinação da razão de teor de água dos grãos de lentilha para as diferentes condições de secagem, foi utilizada a Equação 2.



Onde $\square \square$ é a razão de teor de água; $\square \square$ é o teor de água (base seca); $\square \square \square \square \square \square \square$ é o teor de água inicial (base seca) e $\square \square$ é o teor de água de equilíbrio (base seca).

Os dados obtidos no processo de secagem foram compilados em tabelas no programa Microsoft Excel. Para a analise dos dados experimentais e realização dos ajustes das curvas da cinética foram tomadas como base cinco diferentes modelos matemáticos (Tabela 1) frequentemente usados para representação da secagem de produtos agrícolas.

Tabela 1- Modelos matemáticos utilizados para descrever o processo de secagem.

\begin{tabular}{ll}
\hline Autores & Modelo \\
\hline Page (1949) & $\mathrm{RX}=\exp \left(-\mathrm{Kt}^{\mathrm{n}}\right)$ \\
Midilli, Kucuk \& Yapar (2002) & $\mathrm{RX}=\operatorname{aexp}\left(-\mathrm{Kt}^{\mathrm{n}}\right)+\mathrm{bt}$ \\
Fick (Souza, 2016) & $\mathrm{RX}=\frac{6}{\pi^{2}} \sum_{\mathrm{n}=1}^{\mathrm{s}} \frac{1}{\mathrm{n}^{2}} \exp \left(-n^{2} A t\right)$ \\
Cavalcanti-Mata, Almeida \& Duarte (2006) & $\mathrm{RX}=a_{1} \cdot \exp \left(-\left(k_{1} t\right)^{n_{1}}\right)+a_{2} \exp \left(-\left(k_{1} t\right)^{n_{2}}\right)+a_{a}$
\end{tabular}

Fonte: Autores (2021).

Onde, $\square$ é o tempo de secagem; $\square$ e $\square_{1}$ são constantes de secagem adimensionais; $\square, \square_{1}, \square_{2}, \square_{3}, \square, \square, \square_{1}$ e $\square_{2}$ são coeficientes dos modelos. Além disso, a constante $\square$, foi definida como: $A=\frac{\pi^{2}}{r^{2}} D_{e f}$. 
A difusão em produtos agrícolas, durante a secagem, é um complexo processo, que pode envolver diferentes mecanismos, como a difusão molecular, capilar, na superfície, de vapor e térmica e o fluxo hidrodinâmico (Goneli et al, 2014). A segunda Lei de Fick, descreve que o fluxo de massa por unidade de área é proporcional ao gradiente de concentração de água (Park, Vohnikova \& Brod, 2002). Para a determinação da difusividade efetiva foi utilizada a teoria da difusão, fundamentada na segunda lei de Fick, representada pela Equação 2.

$\frac{\partial x}{\partial t}=\nabla\left(D_{e f} \nabla X\right)$

Onde, $D_{e f}$ é a difusividade efetiva, $\mathrm{m}^{2} \mathrm{~s}^{-1} ; X$ é o teor de água, \% b.s. e $t$ é o tempo, s.

A relação entre a difusividade efetiva e a temperatura de secagem foi descrita através da equação de Arhenius, representada pela Equação 3.

$D_{\text {eff }}=D_{0} \exp \left(-\frac{E}{R T}\right)$

Onde, $D_{0}$ é o coeficiente de difusividade limite, $\mathrm{m}^{2} \mathrm{~s}^{-1}$; E é a energia de ativação, $\mathrm{J} \mathrm{mol}^{-1}$ e $\mathrm{R}$ é a constante universal dos gases, $\mathrm{J} \mathrm{mol}^{-1} \mathrm{~K}^{-1}$.

Linearizando a Equação 3 e aplicando o logaritmo, podemos obter os coeficientes da expressão, como mostrado na Equação 4.

$\ln D_{\text {ef }}=\ln D_{0}-\frac{E}{R} \times \frac{1}{T}$

Considerando materiais homogêneos com coeficiente de difusão constante, a teoria da difusão liquida pode ser representada pela Equação 5.

$\frac{\partial x}{\partial t}=D_{e f}\left(\frac{\partial^{2} x}{\partial r^{2}}+\frac{c}{y} \frac{\partial x}{\partial r}\right)$

Onde c é igual a 0, para corpos planos, 1 para corpos cilíndricos, 2 para corpos esféricos e r distancia radial ou espessura, $\mathrm{m}$.

A partir do desenvolvimento da equação analítica da lei de Fick, obtêm-se as constantes de secagem para modelos empíricos, mostrado na Equação 6.

$k=\frac{R^{2} D_{\text {Lff }}}{R^{2}}$

A entalpia, entropia e a energia livre de Gibbs, foram realizadas utilizando as Equação 7, 8 e 9, respectivamente.

$\Delta H=\Delta E-R T$

$\Delta S=R\left[\ln D_{0}-\ln \left(\frac{k_{b}}{k_{p}}\right)-\ln T\right]$

$\Delta G=\Delta H-T \Delta S$ 
Onde, $\Delta H$ é a entalpia, $\mathrm{J} \mathrm{mol}^{-1} ; \Delta S$ é a entropia, $\mathrm{J}$ mol$^{-1} \mathrm{~K}^{-1} ; \Delta G$ é a energia livre de Gibbs, $\mathrm{J}$ mol ${ }^{-1} ; k_{b}$ é a constante de

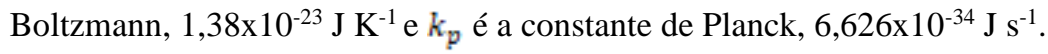

A interpolação dos dados aos modelos matemáticos escolhidos foi realizada com o programa STATISTICA 7.0®. Os modelos foram selecionados tomando-se como parâmetro a magnitude do coeficiente de determinação $\left(\mathrm{R}^{2}\right)$ e o desvio quadrático médio (DQM).

\section{Resultados e Discussão}

A partir dos modelos matemáticos apresentados na Tabela 1 foram realizados ajustes dos dados experimentais com os valores propostos pelo modelo para cada variável utilizada. Os modelos para os ajustes foram escolhidos levando em consideração a análise estatística, para os modelos que possuem os melhores coeficiente de determinação e menores erros da estimativa.

Na Tabela 2, encontram-se os valores estatísticos dos coeficientes de determinação $\left(\mathrm{R}^{2}\right)$ e do desvio quadrático médio (DQM) para os modelos ajustados à cinética de secagem dos grãos de lentilha em diferentes temperaturas.

Tabela 2 - Parâmetros dos modelos analisados, obtidos por meio de regressão não linear para diferentes temperaturas e com teor de água inicial de $25 \%$ base úmida.

\begin{tabular}{|c|c|c|c|c|c|c|c|c|c|}
\hline \multirow{2}{*}{$\begin{array}{c}\text { Modelo } \\
\text { Page }\end{array}$} & \multicolumn{9}{|c|}{ Parâmetros } \\
\hline & & $\mathrm{k}$ & $\mathrm{n}$ & & & & & $\mathrm{R}^{2}(\%)$ & DQM \\
\hline 35 & & 0,0372 & 0,4535 & & & & & 97,90 & 0,0900 \\
\hline 45 & & 0,0451 & 0,4131 & & & & & 98,31 & 0,0445 \\
\hline 55 & & 0,0374 & 0,5360 & & & & & 98,59 & 0,0680 \\
\hline 65 & & 0,0460 & 0,4877 & & & & & 98,25 & 0,0607 \\
\hline Midilli & $\mathrm{a}$ & $\mathrm{k}$ & $\mathrm{n}$ & $\mathrm{b}$ & & & & $\mathrm{R}^{2}(\%)$ & DQM \\
\hline 35 & 1,0493 & 0,0232 & 0,5904 & 0,0001 & & & & 99,74 & 0,0109 \\
\hline 45 & 1,0362 & 0,0305 & 0,5343 & 0,0002 & & & & 99,80 & 0,0054 \\
\hline 55 & 1,0417 & 0,0244 & 0,6604 & 0,0002 & & & & 99,85 & 0,0072 \\
\hline 65 & 1,0342 & 0,0275 & 0,6358 & 0,0002 & & & & 99,85 & 0,0051 \\
\hline Cavalcanti Mata & a1 & $\mathrm{k} 1$ & $\mathrm{n} 1$ & $\mathrm{a} 2$ & $\mathrm{k} 2$ & $\mathrm{n} 2$ & a3 & $\mathrm{R}^{2}(\%)$ & DQM \\
\hline 35 & 0,6723 & 0,0223 & 0,7106 & 2,0170 & $-0,0230$ & 0,0068 & $-1,6893$ & 99,97 & 0,0014 \\
\hline 45 & 1,4571 & $-0,0217$ & 0,0001 & 0,6086 & 0,0395 & 0,6243 & $-1,0566$ & 99,95 & 0,0014 \\
\hline 55 & 0,5734 & 0,0021 & 0,6970 & 0,6241 & 0,0148 & 0,8735 & $-0,1841$ & 99,98 & 0,0009 \\
\hline 65 & 0,6992 & 0,0230 & 0,7677 & 2,0052 & $-0,0008$ & 0,0001 & $-1,6901$ & 99,97 & 0,0010 \\
\hline Fick & & & & $\left(10^{-11} \mathrm{~m}^{2}\right.$ & & & & $\mathrm{R}^{2}(\%)$ & DQM \\
\hline 35 & & & & 1,113 & & & & 99,94 & 0,0045 \\
\hline 45 & & & & 4,622 & & & & 99,92 & 0,0013 \\
\hline 55 & & & & 6,065 & & & & 99,93 & 0,0019 \\
\hline 65 & & & & 8,008 & & & & 99,96 & 0,0004 \\
\hline
\end{tabular}


Fonte: Autores (2021).

Através da Tabela 2 podemos observar que a constante de secagem (k), que segundo Goneli et al. (2009) representa o efeito das condições externas de secagem, obteve um crescimento com o aumento da temperatura do ar de secagem, porém, nas equações de Page e Midilli houve um aumento maior na temperatura de $45^{\circ} \mathrm{C}$, esse aumento pode ser dado por mudanças nas condições externas da secagem, pois foram realizadas com climas e dias diferentes. Para o coeficiente (n), nos modelos de Page e Midilli observou-se um aumento não muito significativo em relação ao aumento da temperatura.

Para todos os tratamentos, os modelos matemáticos ajustados aos dados experimentais apresentaram coeficientes de determinação $\left(\mathrm{R}^{2}\right)$ superiores a $97,90 \%$ e valores de (DQM) inferiores a 0,0900.

Dentre as equações testadas a equação de Fick e a equação de Cavalcanti-Mata, apresentaram maior coeficiente de determinação $\left(\mathrm{R}^{2}\right)$, acima de $99,92 \%$ e menor valores de (DQM), inferior a 0,0045.

As curvas de secagem para cada modelo considerado estão representadas na Figura 2. Em cada figura a curva contínua representa o comportamento esperado com base nas predições dos modelos considerados. A curva preta representa a secagem na temperatura de $35^{\circ} \mathrm{C}$, a curva vermelha para a temperatura de $45^{\circ} \mathrm{C}$, a curva verde para a temperatura de $55^{\circ} \mathrm{C}$ e a curva azul para a temperatura de $65^{\circ} \mathrm{C}$. Pode-se perceber que o melhor ajuste de parâmetros é obtido no modelo CavalcantiMata, com desvio quadrático médio em torno de 0,0012. 
Figura 2 - Valores experimentais e estimados da razão de teor de água em função do tempo para a secagem dos grãos de lentilha às temperaturas de secagem de $35,45,55$ e $65^{\circ} \mathrm{C}$, de acordo com os modelos propostos.

Page

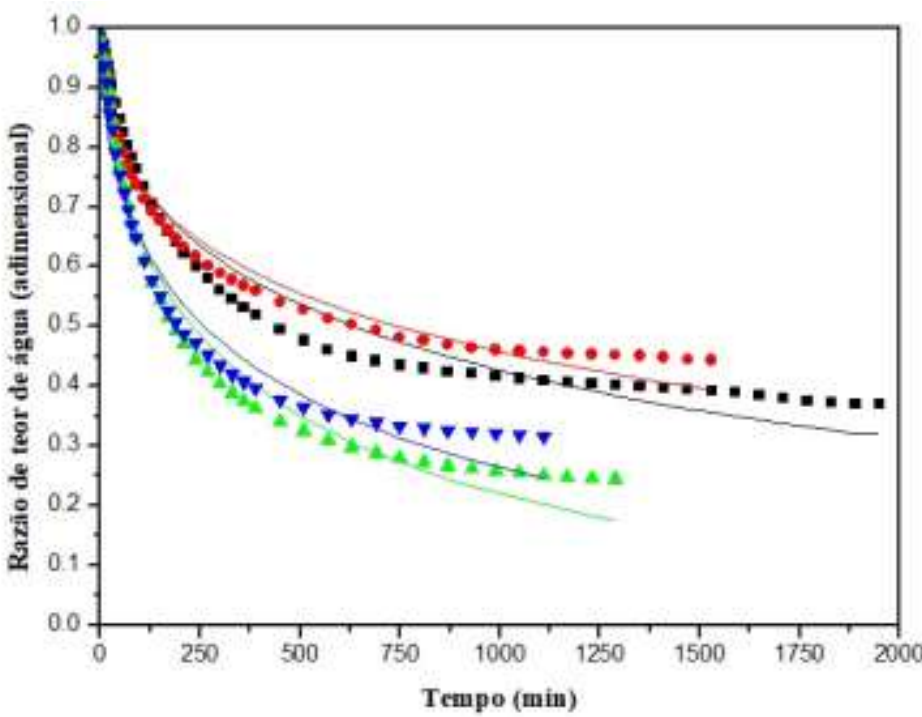

Cavalcanti Mata

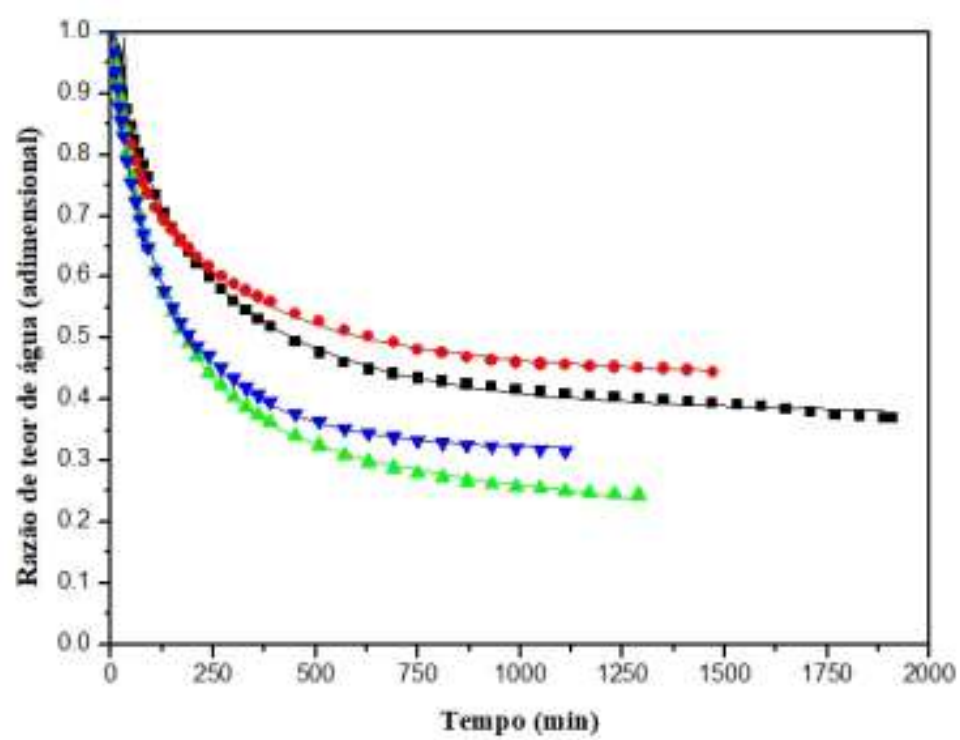

Midilli et al.

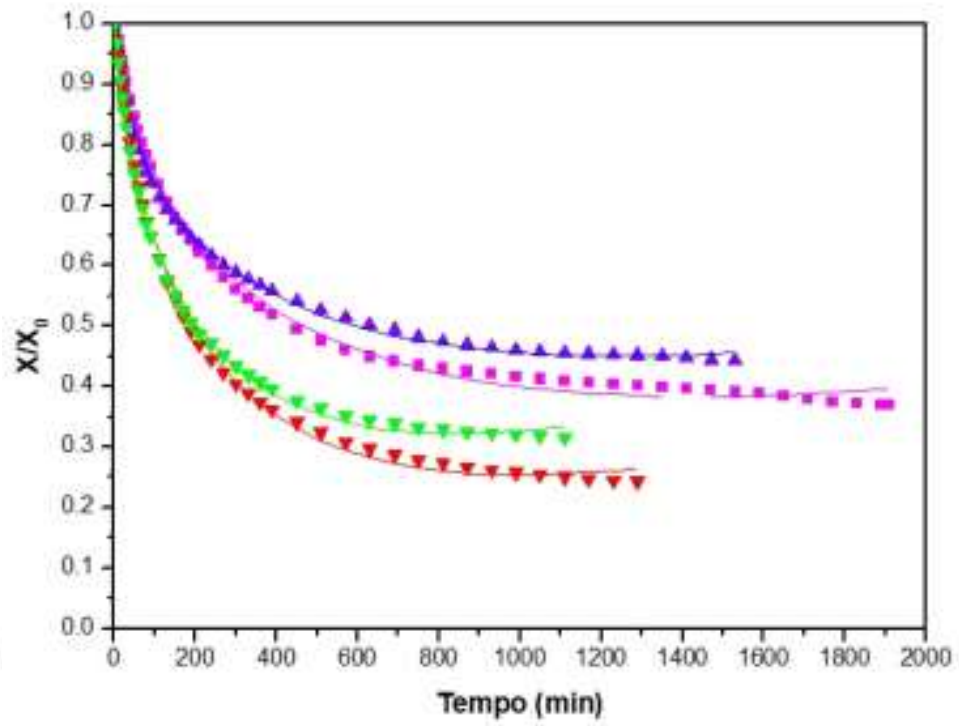

Fick



\section{Secagem a $35^{\circ} \mathrm{C} \quad$ Secagem a $45^{\circ} \mathrm{C}$ \\ Secagem a $55^{\circ} \mathrm{C} \nabla$ Secagem a $65^{\circ} \mathrm{C}$}

Fonte: Autores (2021).

A energia de ativação para a difusão liquida para os grãos de lentilha foi de $23,5 \mathrm{~kJ} \mathrm{~mol}^{-1}$ e está de acordo com valores indicados por Zogzas, Maroulis \& Marinoskouris (1996) para produtos agrícolas, que são entre 12,7 e $110 \mathrm{KJ}^{\mathrm{mol}}{ }^{-1}$. 
As variáveis termodinâmicas obtidas para a cinética de secagem dos grãos de lentilha estão apresentadas na Tabela 3 para o conjunto de temperaturas analisadas e teor de água de $25 \%$ base úmida.

Tabela 3 - Propriedades termodinâmicas para a cinética de secagem dos grãos de lentilha em temperaturas de 35, 45, 55 e 65 ${ }^{\circ} \mathrm{C}$ e teor de água de $25 \%$ base úmida.

\begin{tabular}{|c|c|c|c|}
\hline Temperatura $\left({ }^{\circ} \mathbf{C}\right)$ & $\Delta \mathbf{H}\left({\mathrm{J} . \mathrm{mol}^{-1}}^{-1}\right)$ & 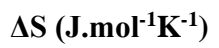 & $\Delta G\left(\mathrm{~J}^{\mathrm{mol}}{ }^{-1}\right)$ \\
\hline 35 & 25938,04 & $-342,84$ & 131583,56 \\
\hline 45 & 25854,90 & $-343,10$ & 135013,27 \\
\hline 55 & 25771,76 & $-343,36$ & 138445,60 \\
\hline 65 & 25688,62 & $-343,61$ & 141880,46 \\
\hline
\end{tabular}

Fonte: Autores (2021).

Podemos observar através da Tabela 3 que a entalpia teve uma pequena diminuição de acordo com a elevação da temperatura, este comportamento está relacionado com o aumento de pressão parcial de vapor de água nos grãos da lentilha com o aumento da temperatura do ar de secagem. Para a entropia ocorreu uma diminuição com o aumento da temperatura do ar de secagem, que pode ser explicado pelo aumento da excitação das moléculas de água de acordo com que a temperatura também aumenta. É possível observar também que a energia livre de Gibbs ocorreu de forma positiva e teve um pequeno aumento com o aumento da temperatura, isso se dar em função de um processo em que o produto recebe energia.

\section{Conclusão}

Conclui-se neste trabalho que dentre os modelos estudados, o modelo Cavalcanti-Mata apresentou o maior coeficiente de determinação $\left(\mathrm{R}^{2}\right)$, igual a 99,98, e menor desvio quadrático médio (DQM), igual a 0,0009, sendo assim o modelo que melhor se ajustou a equação estudada.

A energia de ativação para a difusão liquida para os grãos de lentilha foi de $23,5 \mathrm{~kJ} \mathrm{~mol}^{-1}$.

As propriedades termodinâmicas para a cinética de secagem dos grãos de lentilha foram obtidas de acordo com o esperado, onde a entalpia e entropia diminuíram com de acordo com o aumento da temperatura do ar de secagem e a energia livre de Gibbs foi positiva e aumentou com o aumento da temperatura.

Diante do exposto, ao final desse artigo, sugerimos que os processos de secagem sejam realizados em dias em que o clima esteja semelhante e o mais estável possível, como visto a temperatura ambiente também pode interferir de alguma forma nos resultados. Sugerimos também que sejam estudados outros grãos e outros produtos alimentícios, principalmente produtos regionais, pois é de suma importância aumentar o tempo de vida útil do produto e conseguir um melhor armazenamento, já que o produto desidratado além de possuir uma maior durabilidade, é armazenado mais facilmente e ainda pode ocupar um menor espaço.

\section{Agradecimentos}

Os autores agradecem ao CNPq e CAPES pelo apoio financeiro, a todos os pesquisadores referenciados no texto pela contribuição com seus trabalhos que ajudaram no melhoramento e serviram de base para realização do artigo, e aos professores da Universidade Federal de Campina Grande (UFCG) que contribuíram e deram o suporte necessário. 


\section{Referências}

Bhatty, R. (1990). Cooking Quality of Lentils: The Role of Structure and Composition of Cell Walls. J. Agric. Food Chem., 39(2), $376-383$.

Brasil. (2009). Ministério da Agricultura, Pecuária e Abastecimento. Secretaria de Defesa Agropecuária. Regras para análise de sementes. Brasília: Ministério da Agricultura, Pecuária e Abastecimento, 398.

Brooker, D. B., Bakker-Arkema, F. W., \& Hall, C. W. (1992). Drying and storage of grains and oilseeds. New York: The AVI Van Nostrand Reinhold, 450.

Cavalcanti-Mata, M. E. R. M., Almeida, F. A. C., \& Duarte, M. E. M. (2006). Secagem de sementes. In: Almeida, F. A. C.; Duarte, M. E. M. \& Cavalcanti Mata, M. E. R. M. (Ed.). Tecnologia de armazenamento em sementes. Campina Grande: UFCG, 271-370.

CGC (2014) - Official grain grading guide. Canadian Grain Commission, Winnipeg, Manitoba, Canada http://www.grainscanada.gc.ca/oggg-gocg/18-lentils2014-eng.pdf

FAO (2014). Food and Agriculture Organization of the United Nations. Disponível em: http://www.fao.org.

Goneli, A. L. D., Corrêa, P. C., Afonso Júnior, P. C., \& Oliveira, G. H. H. (2009). Cinética de secagem dos grãos de café descascados em camada delgada. Revista Brasileira de Armazenamento, Viçosa, 11, 64-73.

Goneli, A. L. D., Vieira, M. C., Vilhasanti, H. C. B., Gonçalves, A. A., (2014). Modelagem matemática e difusividade efetiva de folhas de aroeira durante a secagem. e-ISSN 1983-4063-www.agro.br/pat - Pesq. Agropec. Trop., Goiânia. 44(1), 56-64.

Gupta, D., Thavarajah, D., Thavarajah, P., Mcgee, R., Coyne, C. J., \& Kumar, S. (2013). Lentils (Lens culinaris L.), a rich source of folates. Journal of Agricultural and Food Chemistry. 61, 7794-7799.

Joshi, M., Timilsena, Y., \& Adhikari, B. (2017). Global production, processing and utilization of lentil: A review. Journal of Integrative Agriculture, 16(12), 2898-2913.

Martins, J. (2017). Metodologia da pesquisa cientifica. Editora Dowbis, 1, 16-19.

Mayor, L., \& Sereno, A. M. (2004). Modelling Shrinkage During Convective Drying of Food Materials: A Review. J. of Food Engng., 61 , 373-386.

Midilli, A., Kucuk, H., \& Yapar, Z. (2002). A new model for single-layer drying. Drying Technology, Philadelphia, 20(7), 1503-1513 http://dx.doi.org/10.1081/DRT-120005864.

Nascimento, W.M., Vieira, R.F., \& Lima, R.R. (2016). Lentilha. In: Nascimento, W.M. (ED.) Hortaliças Leguminosas. Brasília: Embrapa, 1, $121-146$.

Oliveira, D. E. C. de, Resende, O., Smaniotto, T. A. de S., Siqueira, V. C., \& Neto, C. A. J. (2013). Alterações morfométricas em grãos de soja durante o processo de secagem. Semina: Ciências Agrárias, Londrina-PR, 34(3), 975-984.

Page, C. (1949). Factors influencing the maximum rates of air-drying shelled corn in thin layers. MS Thesis - Purdue University, West Lafayette.

Park, K. J.; Vohnikova, Z.; Brod, F. P. R. (2002). Evaluation of drying parameters and desorption isotherms of garden mint leaves (Mentha crispa L.). Journal of Food Engineering, Londres, 51(3), 193-199.

Resende, O., Corrêa, P., C., Goneli, A., L., D., Botelho, F., M., \& Rodrigues, S. (2008). Modelagem matemática do processo de secagem de duas variedades de feijão (phaseolus vulgaris 1.). Revista Brasileira de Produtos Agroindustriais, 10(1), 17-28.

Roy, F., Boye, J. I., \& Simpson, B. K. (2010). Bioactive proteins and peptides in pulse crops: Pea, chickpea and lentil. Food Research International. 43, 432442. Doi: 10.1016/j.foodres.2009.09.002.

Souza, R. V., Cavalcanti-Mata, M. E. R. M., Duarte, M. E. M., Almeida, R. D., Rosa, M. E. C. (2016). Analise da cinética de secagem em camada fina do arroz vermelho em casca. Revista Brasileira de Produtos Agroindustriais, 18, 517-529.

Tang, J., \& Sokhansanj, S. (1993). Geometric Changes in Lentil Seeds Caused by Drying. J. Agric. Engng. res., 56, $313-326$.

Vohra, K., \& Gupta, V. K. (2012). Pharmacognostic evaluation of Lens culinaris Medikus seeds. Asian Pacific Journal of Tropical Biomedicine 2(3), 12211226 .

Vieira, R.F., Vieira, C., \& Vieira, R.F. (2001). Leguminosas graníferas. Viçosa: UFV, 206.

Zogzas, N., Maroulis, Z. B., \& Marinoskouris, D. (1996). Moisture diffusivity data compilation in foodstuffs. Drying Technology, New York, 14(10), 22252253. 